\title{
Exposure Date Time of Reference Timepoint
}

National Cancer Institute

\section{Source}

National Cancer Institute. Exposure Date Time of Reference Timepoint. NCI Thesaurus.

Code C123997.

The date and time of a specific reference point for the exposure assessment. 